\title{
Energy efficiency in LTE-Advanced networks with relay nodes
}

\author{
Roberto Fantini, Dario Sabella, Marco Caretti \\ Telecom Italia S.p.A. \\ Via G. Reiss Romoli 274, 10148 \\ Turin - Italy \\ roberto.fantini@telecomitalia.it,dario.sabella@telecomitalia.it, marco.caretti@telecomitalia.it
}

\begin{abstract}
- this document analyses the energy efficiency of two relaying schemes that represent a possible way to deploy a relay enhanced network: the two hop scheme and the multicast cooperative scheme. Results exist in literature that provide a model for the theoretical evaluation of the capacity improvement that these solutions can yield, however no analysis is available at present on the energy efficiency of these schemes. This work enhances the existing theoretical models for capacity evaluations, providing a first analysis from an energy efficiency point of view of these two approaches, considering a fully loaded network and the total emitted RF power of the transmitting nodes. Results are provided in terms of the Energy Consumption Index (ECI) of these schemes, a metric proposed in the EARTH project for the assessment of the energy consumption of network solutions. The ECI distribution is obtained and compared with the case of a network deployed without relay nodes, showing that besides the possible improvements in capacity, relays are also candidates as a valuable tool to reduce the energy consumption of a telecommunication network.
\end{abstract}

Keywords-component; relay node; energy efficiency; type 1 relay; type 2 relay; two hop half-duplex relay; multicast cooperative relay; EARTH

\section{INTRODUCTION}

Relays are a quite recent architectural concept for infrastructure networks. Relays are nodes that pick up signals transmitted from a base station to a mobile device and resend an amplified or revised version of the signal to the mobile device. Therefore relays do not have a wired backhaul connection, which makes their deployment cost largely lower. In the last few years, relaying has been studied in the LTEAdvanced study item as a technology that offers the possibility to extend coverage and increase capacity, allowing at the same time more flexible and cost-effective deployment options [1]. Relays nodes (RNs) cover much smaller areas than macro cells, so they have significantly lower transmit power compared to the macro eNodeB (eNB) and are expected to have more in general a power consumption model more efficient. As a consequence Relay Nodes can be considered a promising solution to increase the energy efficiency of a mobile network.

Different approaches have been considered in 3GPP in order to assess the advantages of relaying in terms of capacity and coverage. So far two relay strategies are defined in 3GPP, depending on whether the relay node is part of the donor cell or not: Type 1 and Type 2 relays. A Type 1 relay controls cells of its own, each one with a unique physical-layer cell identity, and it provides all the RRM mechanisms usually offered by a LTE Rel-8 eNodeB, so that it appears to LTE Rel-8 UEs as a normal eNodeB. Type 2 relays are part of the donor cell: they do not create cells of their own, nor they transmit common channels or reference signals typical of a base station. They are transparent to LTE Rel-8 UEs, and their main purpose is to increase network capacity rather than coverage. At least Type 1 relay nodes will be part of LTE-Advanced. A number of contributions exist in 3GPP that provide Shannon Capacity evaluations $[2] \div[6]$, considering both a two hop half-duplex scenario, which can be seen as a Type 1 relay node in 3GPP nomenclature, or advanced schemes like the multicast cooperative scheme, which can be a possible implementation of a Type 2 relay node (see Fig. 1).

This paper will go further in examining the potential of relay enhanced networks, measuring the potential increase in energy efficiency that deploying a certain number of low powered relay nodes can provide.
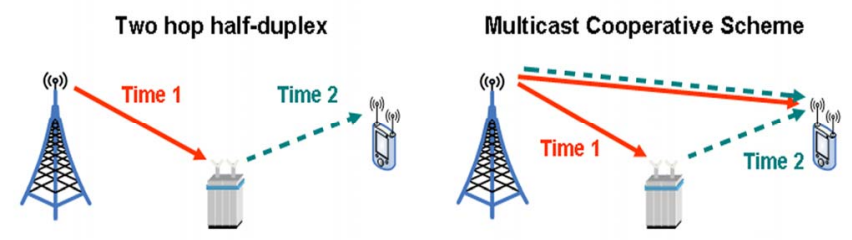

Figure 1. Two hop and multicast cooperative relay schemes

Section II analyzes the energy efficiency of a relay enhanced network in full load condition, taking into account the emitted Radio Frequency (RF) power. Section III provides hints on further considerations that should be addressed in order to align the evaluations here reported to the energy efficiency evaluation framework $\left(\mathrm{E}^{3} \mathrm{~F}\right)$ provided in EARTH project [7], which will be considered in future improvements of this work.

\section{ENERGY EFFICIENCY BASED ON RADIATED POWER}

In this work network performance is analyzed by means of static evaluations in a multi-cell environment, taking into

This work has been carried out within the EARTH (Energy Aware Radio and neTworking tecHnologies) project, which is partly funded by the

European Commission under project FP7-ICT-2009-4-247733-EARTH. 
account average behaviours of the system and assuming that a single user is present in the cell.

The simulated network is represented by a regular hexagonal cellular grid with 19 tri-sectored sites, with inter-site distance (ISD) of 1732 meters, corresponding to Case 3 in 3GPP scenarios [1]. Different deployments can be considered, with a variable number of relays and different possible positions in the cell. In the following study, two Relay Nodes are considered placed near the cell edge. Simulation parameters follow in general the settings in [1], although a static channel is assumed. The main parameters considered are summarized in TABLE I.

TABLE I. SIMULATION PARAMETERS

\begin{tabular}{|l|l|}
\hline \multicolumn{1}{|c|}{ Parameter } & \multicolumn{1}{c|}{ Value } \\
\hline Reference System & LTE FDD $(10 \mathrm{MHz}+10 \mathrm{MHz}), 2.1 \mathrm{GHz}$ \\
\hline Deployment & Hexagonal layout with 3 sector per site. \\
\hline ISD (inter-site distance) & $1732 \mathrm{~m}$ (rural) \\
\hline Relay positions & $(4 / 9 \mathrm{ISD}, \sqrt{3} / 9 \mathrm{ISD})$ \\
& $(4 / 9 \mathrm{ISD},-\sqrt{3} / 9 \mathrm{ISD})$ \\
\hline Antenna System & SISO \\
\hline TX power & eNB $=46 \mathrm{dBm}(40 \mathrm{~W})$ \\
& Relay node $=37 \mathrm{dBm}(5 \mathrm{~W})$ \\
\hline Propagation model & According to $[1]$, case 3 rural, outdoor relays. \\
& No fast fading \\
\hline User distribution & Single user, random with uniform probability \\
\hline Traffic model & Full buffer when scheduled \\
\hline
\end{tabular}

In each point of the cell under investigation, the Shannon capacity available in the direct link (eNB to UE) and that offered considering the relay enhancement has been evaluated, using the various results available in 3GPP for both the two hop half-duplex scenario, or the multicast cooperative scheme $[2] \div[6]$.

For the two hop half-duplex scheme the effective rate, assuming optimal resource division between links that compose the 2-hop connection, is given by:

$$
\left.R_{e f f}\right|_{2-h o p}=\frac{R_{e N B-R N} \cdot R_{R N-U E}}{R_{e N B-R N}+R_{R N-U E}}
$$
[3]:

while for the multicast cooperative scheme it is given by

$$
\left.R_{e f f}\right|_{m-c o o p}=\frac{R_{e N B-R N} \cdot\left(R_{e N B-U E, T 2}+R_{R N-U E}\right)}{\left(R_{e N B-U E, T 2}+R_{R N-U E}\right)+\left(R_{e N B-R N}-R_{e N B-U E, T 1}\right)},
$$

where the rate between node $x$ and $y, R_{x-y}$, can be evaluated knowing the signal to interference ratio of the link $\left(S I N R_{x-y}\right)$ and the available bandwidth $B$ with:

$$
R_{x-y}=B \cdot \log \left(1+\operatorname{SINR}_{x-y}\right) .
$$

Equation (1) is obtained considering that in the two hop scheme the eNB will send an amount of data $b$ to the $\mathrm{RN}$ in a time $T_{1}$, so that

$$
T_{1}=\frac{b}{R_{e N B-R N}} .
$$

The same amount of data will then be delivered from the relay node to the $\mathrm{UE}$ in a time $T_{2}$

$$
T_{2}=\frac{b}{R_{R N-U E}} .
$$

The effective rate will be therefore given by:

$$
R_{e f f}=\frac{b}{T_{1}+T_{2}} .
$$

Knowing this, it is also possible to compute the effective amount of power that will be needed to deliver the data:

$$
\left.P_{e f f}\right|_{2-h o p}=\frac{T_{1}}{T_{1}+T_{2}} \cdot P_{e N B}+\frac{T_{2}}{T_{1}+T_{2}} \cdot P_{R N},
$$

where $P_{e N B}$ and $P_{R N}$ are respectively the eNB and the RN transmitted powers. Using these relations it is possible to derive the Energy Consumption Index (ECI) that provides the energy per bit, defined in EARTH [7] as:

$$
\left.\eta\right|_{2-h o p}=\left.\frac{P_{e f f}}{R_{e f f}}\right|_{2-h o p}=\frac{R_{R N-U E} \cdot P_{N B}+R_{e N B-R N} \cdot P_{R N}}{R_{e N B-R N} \cdot R_{R N-U E}} .
$$

The same approach can be followed for the multicast cooperative scheme, even if in this case at time $T_{2}$ both the eNB and one of the RN are transmitting, therefore:

$$
\left.P_{e f f}\right|_{m-c o o p}=\frac{T_{1}}{T_{1}+T_{2}} \cdot P_{e N B}+\frac{T_{2}}{T_{1}+T_{2}} \cdot\left(P_{R N}+P_{e N B}\right) .
$$

As shown in [2], assuming that the channel is static, in this case $\mathrm{T} 2$ will be equal to:

$$
\left.T_{2}\right|_{m-c o o p}=\frac{b}{R_{e N B-R N}}\left(\frac{R_{e N B-R N}-R_{e N B-U E, T 1}}{R_{R N-U E}+R_{e N B-U E, T 2}}\right) .
$$

Using these expressions the energy consumption index for the multicast cooperative scheme can be derived as:

$$
\begin{aligned}
& \left.\eta\right|_{m-c o o p}=\left.\frac{P_{e f f}}{R_{e f f}}\right|_{m-c o o p}=\frac{R_{1} \cdot P_{N B}+R_{2} \cdot P_{R N}}{R_{e N B-R N} \cdot\left(R_{R N-U E}+R_{e N B-U E, T 2}\right)} \\
& R_{1}=R_{R N-U E}+R_{e N B-R N}+R_{e N B-U E, T 2}-R_{e N B-U E, T 1} \\
& R_{2}=R_{e N B-R N}-R_{e N B-U E, T 1}
\end{aligned}
$$

Using the previous formulas and 3GPP parameters defined for a specific scenario (urban, sub-urban, rural), it is possible to build maps showing in each point of the cell the available SINR, capacity and expected energy efficiency. These results can be compared with similar results obtained with a classical deployment without relays.

As shown in Fig. 2, adding two RNs can improve the SINR in the area near the RNs, and this allows to increase also capacity in the surrounding area, as highlighted in Fig. 3, where the improvements of capacity near the relay nodes for the two hop half duplex and multicast cooperative schemes are visible. Starting from these results it is possible to collect the capacity distribution across the cell, and compare it with similar results obtained in the case of a network without relay nodes. 

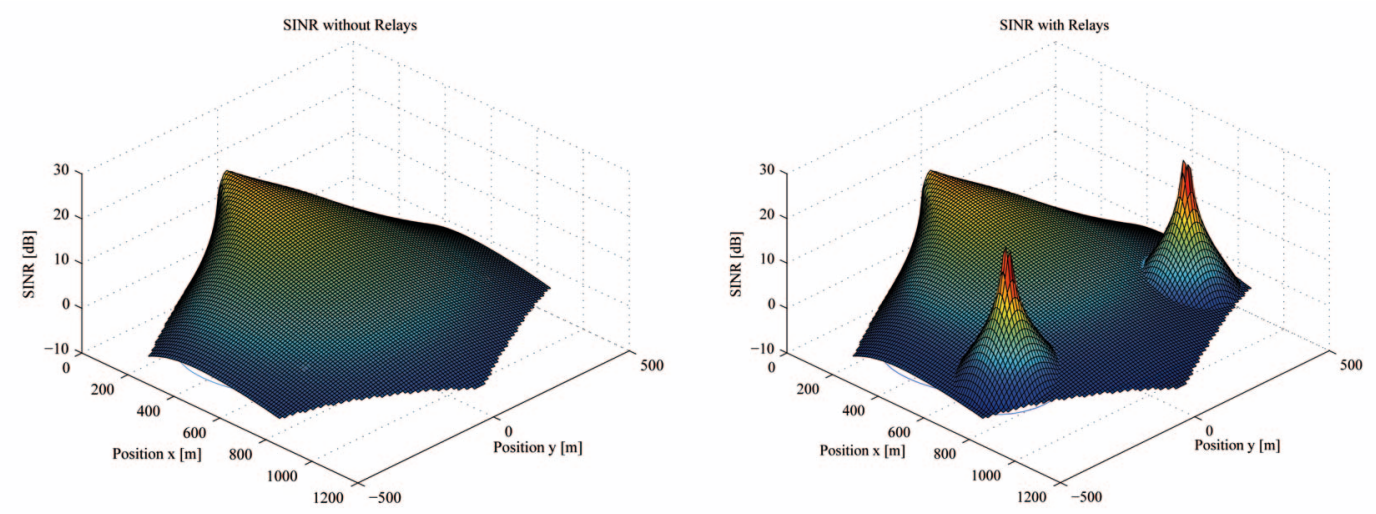

Figure 2. Signal to interference ratio in the cell area without and with two RNs.
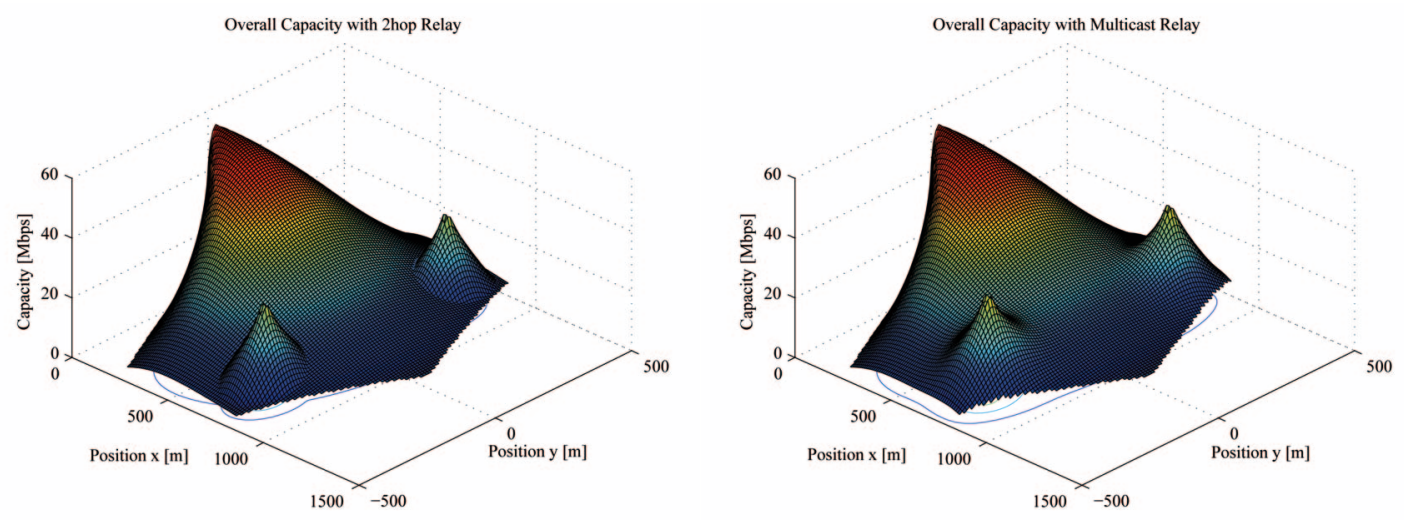

Figure 3. Capacity in the cell area with 2-hop or multicast cooperative schemes.

According to the simulations, even if the multicast cooperative scheme can provide slightly better improvement in capacity (see Fig. 4), the two hop scheme is more energy efficient, as shown in Fig. 5. In particular, from the distributions in Fig. 5 it can be found that the 2 hop scheme in the simulated scenario has on average an energy saving of $15.6 \%$ respect to the case without relays, while in the multicast cooperative scheme the energy saving is slightly lower, and equal to $8.5 \%$.

The improvement in energy efficiency is first of all due to the improvement in capacity offered by both schemes, which can be seen clearly in Fig.4 comparing the results of the relay schemes with those obtained with a network without relays. Distributions associated with relays shifts to the right in the figure, meaning that higher levels of throughput are more probable. However this capacity improvement is obtained through additional power transmitted by the RN, so the actual energy per bit will depend also on how this power is used in these two schemes.

In the two hop scheme the transmission is completed in two steps: during the first step from the BS to the RN only the high power emitted by the BS is used, but the link is usually in good radio condition and it offers a high capacity, so that this first transmission has higher energy efficiency than what would be experienced in transmitting directly to the UE. During the second step, transmission is performed from the RN to the UE, and in this case only the relatively low power emitted by the $\mathrm{RN}$ is consumed, so that also for this step energy efficiency can be high. In the end, in the area served by the relay, the combination of these two steps provides better energy consumption indexes than the transmission arriving directly from the eNB.

Note that the two hop gain can be obtained only in the area served by the relay, therefore the previously reported $15.6 \%$ overall gain for the two hop scheme, which is obtained averaging the results in the whole cell, corresponds actually to a far higher gain in the relay area, in the order of $73.2 \%$.

In the multicast cooperative case on the other hand, the energy consumption improvement is mainly due to the higher capacity that can be offered by this scheme. In this case in fact, the BS is transmitting all of the time and during the cooperation phase there will be also the additional power due to the relay transmission that increases the overall power consumption. 
For this reason the overall energy efficiency is lower if compared to the 2 hop scheme, even if from a capacity point of view the multicast scheme performed better.

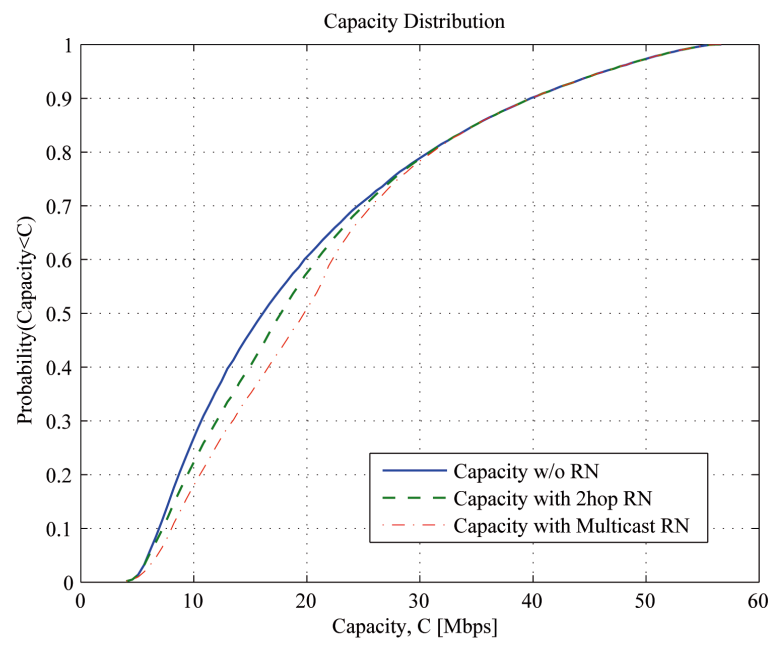

Figure 4. Shannon Capacity cumulative distribution

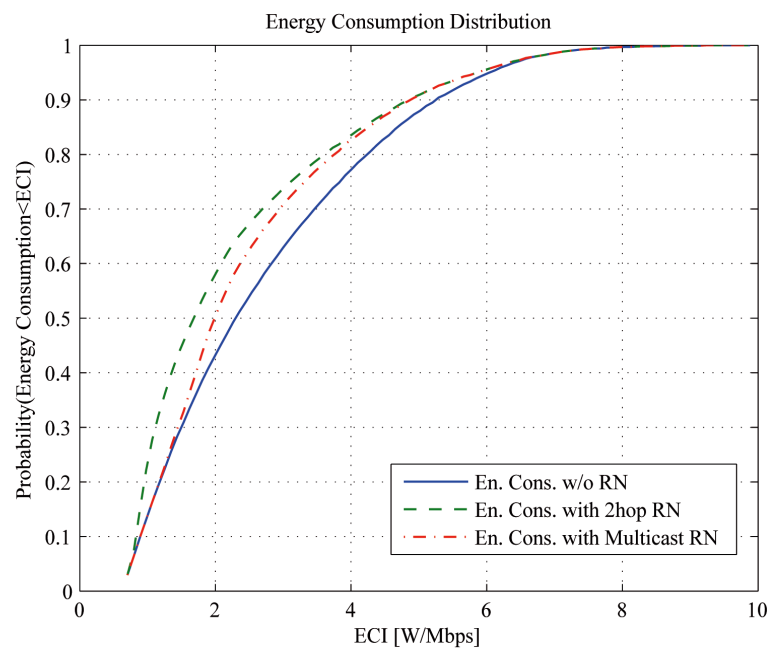

Figure 5. Energy Consumption Index cumulative distribution

\section{FUTHER WORKS}

Results presented in this work provide a first positive indication on the possibility to use relays as a tool to increase the energy efficiency in a communication network. However this study should be considered as a first step in the complete energy efficiency assessment of relays.

These results in fact are obtained considering only the consumption due to the radiated RF power of the transmitting nodes, and are relative to the specific case of full load, assuming therefore that all the radio frequency resources (in power and bandwidth) are always used.
In [7] the EARTH project has provided power models for different kind of base station (macro, micro, pico and femto), showing that the total power consumed by a transmission node is linked to the RF power, but is usually also much higher. In particular it has been shown that in general the relations between relative RF output power and BS power consumption of these configurations are nearly linear, but that the radiated power is only a small fraction of the total amount of consumed power.

At present however discussion on a realistic power models for relay nodes is still ongoing in EARTH, since no commercial solution is available. Still it is clear that in order to assess completely the energy efficiency of a relay enhanced network it is necessary to include in the simulation framework a power model that captures the whole power consumed by all transmitting nodes.

Another important aspect is that energy consumption is strongly linked with the traffic load in the cell. For this reason an analysis that only considers full load scenario may lead to results that do not capture properly the actual energy consumption, since most of the time real networks are not fully loaded.

Moreover, as highlighted in [7], a small scale simulation where only a certain deployment scenario (urban, sub-urban or rural) is considered fails to capture the complexity of a countrywide network, where a mix of deployment is usually found.

Therefore as proposed in the EARTH project, for the global assessment of network energy efficiency, all these aspects need to be included in the simulator, using proper power models, long-term traffic variations, and the mix of deployment scenarios that quantify the area covered by cities, suburbs, highways and villages, as depicted in [7].

\section{CONCLUSIONS}

This work analyses the energy efficiency of two possible schemes that have been discussed as a possible way to deploy a relay enhanced LTE-Advanced network, the two hop scheme (Type 1 relay) and the multicast cooperative scheme (possible implementation of a Type 2 relay).

Results are provided considering small scale simulations in a multi-cell environment, in full load conditions and by measuring only the radiated power and the relationship with corresponding obtained cell throughput. In particular it has been shown that energy savings up to $15.6 \%$ are possible in the two hop schemes, and up to $8.5 \%$ are possible with the multicast cooperative scheme. So, even if the multicast cooperative scheme can provide higher capacity in the cell, a two hop scheme proved to be more energy efficient.

These results are obtained averaging gains in the whole cell, therefore they actually corresponds to higher gain in the relay covered areas. In general, they can be considered as a first indication of the potentiality of relay nodes as a tool to increase the energy efficiency in a network, that paves the way to further studies aligned to the EARTH E3F framework. 


\section{ACKNOWLEDGMENT}

This work has been performed in the framework of the FP7 project FP7-ICT-2009-4-247733-EARTH, which is partially funded by the European Union.

\section{REFERENCES}

[1] 3GPP TR 36.814 v1.5.1 (2009-12), "Further Advancements for EUTRA, Physical Layer Aspects”.

[2] 3GPP R1-083205 "Application of L2 Relay in an Interference Limited Environment for LTE-A," TSG RAN WG1 \#54, Jeju, South Korea, 2008.

[3] 3GPP R1-091423 "Comparison of Type 1 Relay and L2 Cooperative Relay,” TSG-RAN WG1 \#56bis, Seoul, South Korea, 2009.

[4] 3GPP R1-090073 “A system simulation study of downlink L2 relay network,” TSG RAN WG1 \#55bis, Ljubljana, Slovenia, 2009.

[5] 3GPP R1-100353 "Comparing In-band vs. Out-band Relays in coverage limited scenario,” TSG RAN WG1 \#59bis, Valencia, Spain, 2010.

[6] R1-084354 "Considerations on the Relay Maximum Transmit Power," TSG RAN WG1 \#55, Prague, Czech Republic, 2008.

[7] EARTH project, "D2.3 - Energy efficiency analysis of the reference systems, areas of improvements and target breakdown," https://www.ictearth.eu/publications/publications.html 